\title{
Spherical Fibonacci Point Sets for Illumination Integrals
}

\author{
R. Marques ${ }^{1}$, C. Bouville ${ }^{2}$, M. Ribardière ${ }^{2}$, L. P. Santos ${ }^{3}$ and K. Bouatouch ${ }^{2}$ \\ ${ }^{1}$ INRIA Rennes, France \\ ${ }^{2}$ IRISA Rennes, France \\ ${ }^{3}$ Universidade do Minho, Braga, Portugal
}

\begin{abstract}
Quasi-Monte Carlo (QMC) methods exhibit a faster convergence rate than that of classic Monte Carlo methods. This feature has made QMC prevalent in image synthesis, where it is frequently used for approximating the value of spherical integrals (e.g., illumination integral). The common approach for generating QMC sampling patterns for spherical integration is to resort to unit square low discrepancy sequences and map them to the hemisphere. However such an approach is suboptimal as these sequences do not account for the spherical topology and their discrepancy properties on the unit square are impaired by the spherical projection. In this article we present a strategy for producing high quality QMC sampling patterns for spherical integration by resorting to spherical Fibonacci point sets. We show that these patterns, when applied to illumination integrals, are very simple to generate and consistently outperform existing approaches, both in terms of Root Mean Square Error (RMSE) and image quality. Furthermore, only a single pattern is required to produce an image, thanks to a scrambling scheme performed directly in the spherical domain.
\end{abstract}

\section{Introduction}

Among all the methods which have been proposed to speed up Monte Carlo integration for rendering, Quasi-Monte Carlo (QMC) methods play an important role as they allow improving the convergence rate as well as controlling the error noise perception. The principle is to use more regularly distributed sample sets (i.e., with some determinism) than the crude random sample sets associated with Monte Carlo integration.

QMC integration is now extensively used in computer graphics (see e.g., [SEB08]). Keller has shown in [Kel12] that QMC techniques can be applied in a consistent way to deal with a wide range of problems (anti-aliasing, depth of field, motion blur, spectral rendering,...). However, few applications have been reported in the literature specifically addressing hemispherical sampling with a view of computing the illumination integral. Unlike the unit square sampling case, no explicit construction of optimal point sets for spherical sampling is known and generally the spherical point sets are generated by lifting point sets from the unit square to the unit sphere through an equal-area transform. Although such point constructions are not proved to be optimal, recent results from the numerical analysis literature suggest that both $(0,2)$-sequences and Fibonacci lattices lifted to the sphere are quite close to optimality in terms of discrepancy [ABD12,BD11]. Nevertheless their performance is not exactly equivalent: several authors have shown that spherical Fibonacci lattices are particularly well-suited to sphere sampling compared to other low-discrepancy point sets. Furthermore, similar point structures arise spontaneously in nature so as to implement a best packing strategy on the sphere (e.g., packing of seeds in the sunflowers head [Vog79]), a clear indication that these structures have intrinsically good spherical uniformity properties.

In this paper, we introduce theoretical aspects on QMC spherical integration that, to the authors knowledge, have never been used in the graphics community. In concrete terms, we define worst case integration error (w.c.e.), spherical cap discrepancy (s.c.d.) and an inter-samples distancebased energy metric $E_{N}$, which allows to assess the quality of a spherical samples set for spherical integration.

The second and major contribution of this work is the introduction of the Fibonacci point sets for spherical quadrature, based on previous works [HN04, SJP06]. We compare the quality of Fibonacci point sets for estimating the illumination integral with that of state-of-the-art QMCcompliant point set distributions such as blue noise [dGBOD12], Larcher-Pillichshammer point sets [LP01] and the 

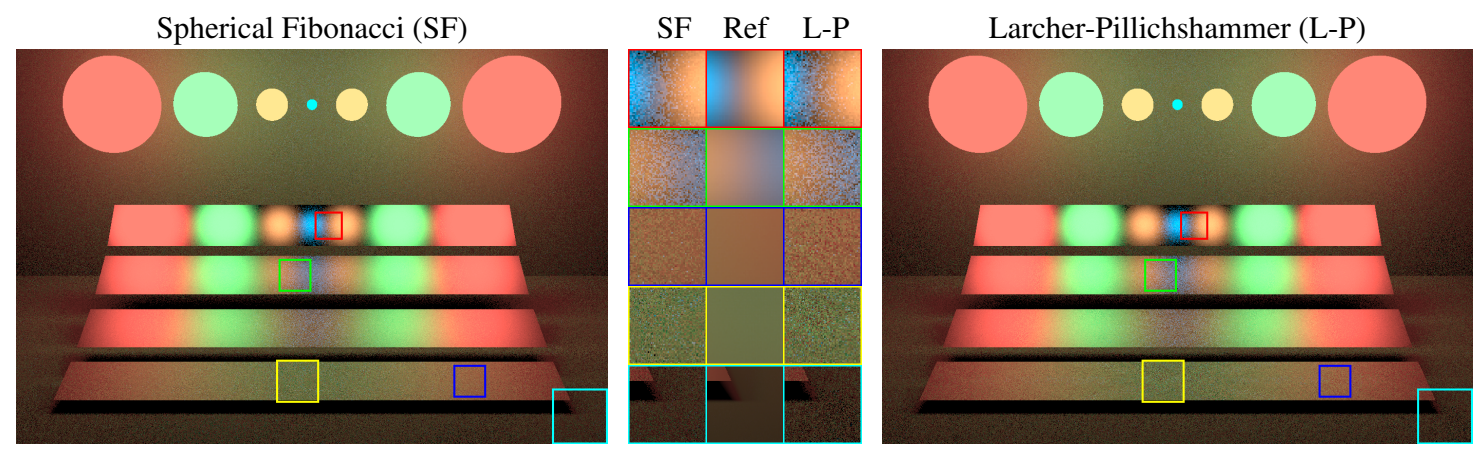

Figure 1: This figure shows how spherical Fibonacci $(S F)$ point sets behave for an incident radiance function covering a wide range of frequencies and materials of different glossinesses. Direct lighting of different plates with light sources of varying size using SF (left) and Larcher-Pillichshammer (L-P, right) point sets. The L-P point sets have been projected using the Lambert cylindrical projection. The Phong shininess coefficient $n$ of each of the plates is 10, 50, 80 and 200 from bottom to top respectively, while the background is perfectly diffuse. The RMSE of the image rendered using the L-P points is $7.55 \%$ higher. The maximum quadratic error per pixel is 0.39 for $S F$ and 0.71 for L-P.

popular Sobol $(0,2)$-sequence [Sob67]. We show that the Fibonacci point sets consistently outperform those methods and that the improvement is, in general, remarkable in terms of RMSE value and percentage of rays saved for the same RMSE quality. The noise perception in the resulting images is also reduced. Furthermore, the generation of the Fibonacci point sets is much simpler than the other tested methods, and a single sequence is needed to synthesize an image.

The rest of this paper is structured as follows. In the next section, we introduce theoretical concepts regarding spherical integration using QMC and present the related work. It is followed by a detailed description of the Fibonacci spherical point sets. In section 4 , we specify how we have implemented BRDF sampling in the context of QMC integration and make explicit the interest in generating high quality spherical distributions for this particular case. Sections 5 and 6 present the benefits of using Fibonacci point sets compared to a Sobol sequence, blue noise point sets and the LarcherPillichshammer points. We finish with a conclusion and future work.

\section{Background}

\subsection{QMC spherical integration}

The goal of QMC integration is to find sampling patterns that yield a better order of convergence than the $O\left(N^{-1 / 2}\right)$ rate obtained with purely random distributions. In the case of QMC integration over the unit square $[0,1]^{2}$, it is wellknown that the best theoretical rate of convergence of the worst case error is $O\left(N^{-1} \sqrt{\log N}\right)$ (see e.g., [BD11]). To find point set constructions that approximate this optimal rate of convergence, the star-discrepancy is often used as a criterion to characterize the uniformity of the point distribution (the connection between this criterion and the worst case error is given by the Koksma-Hlawka inequality [Nie88, BD11]). Moreover, a point set construction is called a low-discrepancy sequence when its unit square discrepancy convergence rate towards 0 is of order $O\left(N^{-1}(\log N)^{2}\right)$.

Unlike the unit square case, QMC rules for numerical integration over the unit sphere $\mathbb{S}^{2}$ in $\mathbb{R}^{3}$ are less known to the graphics community. Therefore, a brief presentation of important results on this subject will be given in the following of this section.

A set of sampling directions $\left\{\omega_{1, N}, \ldots, \omega_{N, N}\right\}$ defined as points on the unit sphere $\mathbb{S}^{2}$ is appropriate for Monte Carlo integration if it is asymptotically uniformly distributed, that is if

$$
\lim _{N \rightarrow \infty} \frac{1}{N} \sum_{j=1}^{N} f\left(\omega_{j, N}\right)=\frac{1}{4 \pi} \int_{\mathbb{S}^{2}} f(\omega) d \Omega(\omega)
$$

is true for every function $f(\omega)$ on the sphere $\mathbb{S}^{2}, \Omega$ being the surface measure on $\mathbb{S}^{2}$. Similarly to the unit square case, this property is equivalent to:

$$
\lim _{N \rightarrow \infty} \frac{\operatorname{Card}\left\{j: \omega_{j, N} \in C\right\}}{N}=\frac{\Omega(C)}{4 \pi}
$$

for every spherical cap $C$ with area $\Omega(C)$ [KN06]. Informally speaking, Eq (2) means that a spherical cap of any area has its fair share of points as $N \rightarrow \infty$. Among all sampling patterns complying with this definition, we are interested in point sets $P_{S}=\left\{\omega_{1, N}, \ldots, \omega_{N, N}\right\} \subseteq \mathbb{S}^{2}$ such that the worst case integration error (w.c.e.)

$$
\text { w.c.e. : }=e\left(P_{s}\right)=\sup _{f}\left|\frac{1}{N} \sum_{j=1}^{N} f\left(\omega_{j, N}\right)-\frac{1}{4 \pi} \int_{\mathbb{S}^{2}} f(\omega) d \Omega(\omega)\right|
$$

achieves the best rate of convergence as $N \rightarrow \infty$. This is equivalent to finding the point sets $P_{s}$ which minimize the 
spherical cap discrepancy (s.c.d.) defined as follows:

$$
\text { s.c.d. : }=D\left(P_{s} ; C\right)=\sup _{C \subseteq \mathbb{\$}^{2}}\left|\frac{\operatorname{Card}\left\{j: \omega_{j, N} \in C\right\}}{N}-\frac{\Omega(C)}{4 \pi}\right|
$$

where the supremum is extended over all spherical caps $C \in \mathbb{S}^{2}$. The mathematical relationship between w.c.e. and s.c.d. is more complex than in the unit square case as explained in [BD11, BSSW12]. Minimizing the s.c.d. is still equivalent to minimizing the w.c.e. However, both criteria only follow the same $O\left(N^{-3 / 4}\right)$ rate of convergence towards 0 if $f$ fulfills a specific smoothness criterion. Roughly speaking, it must be at least a $C_{0}$ continuous function. In such a case, in application of the Stolarsky's invariance principle, the w.c.e. is proportional to the distance-based energy metric $E_{N}[\mathrm{BD} 11, \mathrm{BSSW} 12]$ given by

$$
E_{N}\left(P_{s}\right)=\left(\frac{4}{3}-\frac{1}{N^{2}} \sum_{j=1}^{N} \sum_{i=1}^{N}\left|\omega_{i}-\omega_{j}\right|\right)^{\frac{1}{2}},
$$

which means that minimizing the w.c.e. is equivalent to maximizing the sum of distances term $\sum_{j=1}^{N} \sum_{i=1}^{N}\left|\omega_{i}-\omega_{j}\right|$ while keeping the property of asymptotically uniform distribution. $E_{N}$ can also be interpreted as an optimal spherical packing criterion [SK97].

The order of convergence of the w.c.e. can be higher than $O\left(N^{-3 / 4}\right)$ if the order of continuity of the integrand is higher than $C_{0}$, but this depends on the points construction algorithm since some are more capable of taking advantage of smooth functions than others as explained in [BSSW12].

The s.c.d. order of convergence cannot be better than $O\left(N^{-3 / 4}\right)$ but there surely exist point sets for which the order of convergence is better than $O\left(N^{-3 / 4} \sqrt{\log N}\right)$ [Bec84], in which case these configurations are said to be lowdiscrepancy sequences. Note that this order of convergence is lower than the $O\left(N^{-1}(\log N)^{2}\right)$ rate of low-discrepancy sequences in the $[0,1]^{2}$ unit square.

In contrast with the unit square case, no explicit direct construction of low-discrepancy sequences on the unit sphere is known. That is why QMC sequences on $\mathbb{S}^{2}$ are generally produced by lifting a $[0,1]^{2}$ low discrepancy point set to $\mathbb{S}^{2}$ through an equal-area transform. An alternative to this approach consists in generating the patterns directly on the sphere according to an extremal energy criterion [SK97]. Among the patterns with good $E_{N}$ properties, spherical Fibonacci point sets (or equivalently generalized spiral points) are particularly well-suited to QMC integration over the sphere as shown in [HNO4], hence our interest in applying them for illumination integral computation.

\subsection{Related work}

The use of low discrepancy sequences is widespread in computer graphics [KPR12]. Their goal is to improve the convergence rate of the integral estimate by using sample sets which minimize a discrepancy criterion. Among the most popular low-discrepancy sequences is the Sobol's $(0,2)$-sequence [Sob67] which guarantees both minimum distance and stratification criteria in each successive set of $b^{m}$ samples, where $b$ is the base of the sequence. Lower unit square discrepancy values can be obtained using the Larcher-Pillichshammer point sets [LP01], however these points cannot be generated by an (infinite) sequence. Moreover, Kollig et al. [KK02] showed that both sequences can be easily scrambled to decorrelate directions for neighboring pixels, thus avoiding artifacts without sacrificing the discrepancy and stratification properties.

An alternative approach for producing uniform point set distributions on a unit square is to use a blue noise generator [LD08]. This class of point set generators produces high quality uniform (yet unstructured) distributions which try to approach the spectral characteristics of Poisson disk distributions. The goal is to concentrate the noise in high frequencies where it is less visible. The resulting distributions exhibit better uniformity properties when compared to $(0,2)$-sequences, but this is achieved at a higher computational cost. Recent works have focused on efficient generation of high quality blue noise patterns $\left[\mathrm{CYC}^{*} 12\right.$, dGBOD12, EPM*11, Fat11], among which the state-of-the-art is currently given in [dGBOD12].

The unit square-based distributions generated by the methods described above must be lifted to the $\mathbb{S}^{2}$ sphere using an equal-area projection so as to be used for (hemi)spherical integration. Such projections preserve the property of asymptotic distribution uniformity, but not the samples distance. As discrepancy and w.c.e. directly depend on the distance between samples (see Eq. (3)), the resulting sets become suboptimal for (hemi)spherical sampling.

An explicit spherical construction of point sets with small s.c.d. has been proposed in [LPS86], but recently a better order of convergence has been reported by lifting $(0,2)$ sequences and Fibonacci lattices from the unit square $[0,1]^{2}$ to the $\mathbb{S}^{2}$ sphere [ABD12, BD11]. Both resulting sampling patterns exhibit good discrepancy properties in the spherical domain. Nevertheless their performance is not exactly equivalent as shown by other authors [Nye03, Gon10] which conclude that Fibonacci lattices are more efficient.

Throughout this paper, we will use a spherical Fibonacci lattice implementation based on [SJP06]. We will show that this algorithm is simpler and more efficient than the Sobol $(0,2)$-sequence [Sob67], the state of the art blue noise [dGBOD12] and the Larcher-Pillichshammer point sets [LP01] when the goal is spherical sampling.

\section{Spherical Fibonacci point sets}

Our goal in this section is to explain how Fibonacci lattices are generated and why such point constructions are well-suited to spherical sampling. In the following, we introduce spherical Fibonacci point sets through a lifting pro- 
cedure from the unit square to the unit sphere. We have chosen this procedure since it allows establishing a connection with traditional QMC point constructions defined over the unit square. However the same point sets can be equivalently derived on the sphere or on a disk as shown in [SK97] or [Vog79] respectively.

A Fibonacci lattice in the unit square is a set $Q_{m}$ of $F_{m}$ points $(x, y)$ defined as follows [NH94]:

$$
\left.\begin{array}{l}
x_{j}=\left\{j \frac{F_{m-1}}{F_{m}}\right\} \\
y_{j}=\frac{j}{F_{m}}
\end{array}\right\} 0 \leq j<F_{m},
$$

where $F_{m-1}$ and $F_{m}$ are the two last numbers of a sequence of $m+1$ Fibonacci numbers [GKP94] given by the recurrence equation $F_{m}=F_{m-1}+F_{m-2}$ for $m>1 . F_{0}=0, F_{1}=1$, and $\{x\}=x-\lfloor x\rfloor$ denotes the fractional part for non-negative real numbers $x$. By directly lifting this lattice to the unit sphere with the cylindrical Lambert map, we obtain the following point set [Sve94, HN04]:

$$
\begin{aligned}
\theta_{j} & =\arccos \left(1-2 j / F_{m}\right) \\
\phi_{j} & =2 \pi\left\{j \frac{F_{m-1}}{F_{m}}\right\}
\end{aligned}
$$

where $\theta_{j}$ and $\phi_{j}$ are the polar and azimuthal angles respectively of a lattice node $\omega_{j}$. As the Fibonacci ratio $F_{m} / F_{m-1}$ quickly approaches the golden ratio $\Phi=(1+\sqrt{5}) / 2$ as $m$ increases [GKP94], we can write:

$$
\lim _{m \rightarrow \infty} \phi_{j}=2 j \pi \Phi^{-1}
$$

due to the periodicity of the spherical coordinates. Hence, setting $F_{m}=N$, the coordinates of an $N$-point spherical Fibonacci set are given by:

$$
\left.\begin{array}{rl}
\theta_{j} & =\arccos (1-2 j / N) \\
\phi_{j} & =2 j \pi \Phi^{-1}
\end{array}\right\} 0 \leq j<N
$$

Note that in this case, $\mathrm{N}$ needs not be a Fibonacci number anymore, which allows generating point sets with an arbitrary number of points. The resulting point sets are no longer lattices when projected back in the original $(x, y)$ plane since $\Phi$ is irrational. Therefore, from now on, these point sets will be called spherical Fibonacci (SF) point sets. Letting $z_{j}$ denote the $z$ coordinate of point $j$, we have:

$$
z_{j}=\cos \theta_{j}=1-2 j / N
$$

which means that the $z$ coordinates of the lattice nodes are evenly spaced. Such an arrangement divides the sphere into equal-area spherical "rings" due to the area-preserving property of the Lambert map [Gon10], each "ring" containing a single lattice node. Swinbank et al. [SJP06] slightly improved the point set used in [HN04] by introducing an offset of $1 / N$ to the $z_{j}$ coordinates (i.e. half the $z$ coordinate spacing) to achieve a more uniform distribution near the poles. Then we have:

$$
\left.\begin{array}{l}
\theta_{j}=\arccos \left(1-\frac{2 j+1}{N}\right) \\
\phi_{j}=2 j \pi \Phi^{-1}
\end{array}\right\} 0 \leq j<N
$$

As observed in [Gon10], the same point set can be produced using $\Phi^{-2}=(3-\sqrt{5}) / 2$ instead of $\Phi^{-1}$. The $\phi_{j}$ angles will then be multiples of the golden angle $\pi(3-\sqrt{5})$. More details on the properties of the spherical Fibonacci point set can be found in [SJP06, Gon10]. In particular, this point set can also be generated by projecting a Fermat spiral on a sphere, also known as the cyclotron spiral. This arrangement can also be found in nature (e.g., the packing of seeds on the sunflowers head [ $\operatorname{Vog} 79])$, a clear indication of its near-optimality w.r.t. the distance based energy metric $E_{N}$ (Eq. (3)). Other theoretical approaches proposed in the literature lead to similar arrangements (e.g., [SK97]).

In the case of illumination integrals (see Eq. (5)), the integration domain is not the sphere, but the hemisphere $\Omega_{2 \pi}$, where the vertical axis $z$ is aligned with the surface normal. By modifying Eq. (4), an $N$-point hemispherical SF point set will then be defined as follows:

$$
\left.\begin{array}{rl}
\theta_{j} & =\arccos \left(z_{j}\right) \\
\phi_{j} & =2 j \pi \Phi^{-1}
\end{array}\right\} 0 \leq j<N
$$

where the $z_{j}=\left(1-\frac{2 j+1}{2 N}\right)$ are the $z$-coordinates of the points on the hemisphere. Such a point set can be very easily generated using the pseudo-code presented in Alg. 1.

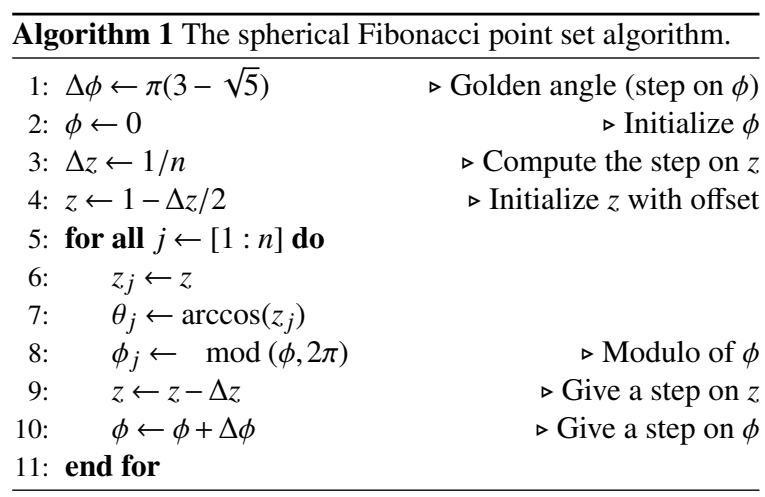

Image synthesis involves the computation of many illumination integrals. Using the same point set for computing all illumination integrals results in visible patterns in the rendered images. To avoid this problem the sample sets must be scrambled at each illumination integral evaluation. We used a scrambling strategy of the SF sampling pattern which is made directly in the spherical domain by rotating them about the $\mathrm{z}$-axis with a random angle uniformly distributed over $[0,2 \pi]$. This method has proved to be efficient as will be seen in the results, as no low frequency patterns can be seen. This method has the advantage of preserving the intersamples distances and thus the energy $E_{N}$. When using the Lambert cylindrical projection, a rotation about the $z$ axis on the sphere is equivalent to a Cranley-Patterson [CP76] rotation along the $x$ axis in unit square. 


\section{QMC for illumination integrals}

To render an image of a scene, the illumination integral must be computed at each point of visible surfaces. This integral gives the reflected radiance $L_{o}\left(\omega_{o}\right)$ at a given visible point and can be expressed as follows:

$$
L_{o}\left(\omega_{o}\right)=\int_{\Omega_{2 \pi}} L_{i}\left(\omega_{i}\right) \rho\left(\omega_{i}, \omega_{o}\right)\left(\omega_{i} \cdot \mathbf{n}\right) d \Omega\left(\omega_{i}\right)
$$

where $\rho\left(\omega_{i}, \omega_{o}\right)$ is the BRDF, $\mathbf{n}$ is the surface normal at the shading point and $\Omega_{2 \pi}$ is the hemisphere of unit radius, the main axis of which is aligned with $\mathbf{n}$. The incident direction $\omega_{i}$ and the direction of observation $\omega_{o}$ are considered as points on the unit hemisphere $\Omega_{2 \pi}$. A straightforward application of Eq. (1) would consist in computing an estimate of $L_{o}\left(\omega_{o}\right)$ by averaging samples of the integrand of Eq. (5) with uniformly-distributed sampling points on $\Omega_{2 \pi}$. Such an approach would be quite inefficient since the product $\rho\left(\omega_{i}, \omega_{o}\right)\left(\omega_{i} \cdot \mathbf{n}\right)$ is generally close to zero in a large part of the integration domain. In classic Monte Carlo method, a common solution is to distribute the samples according to a pdf proportional to $\rho\left(\omega_{i}, \omega_{o}\right)\left(\omega_{i} \cdot \mathbf{n}\right)$. In the QMC deterministic context, as probabilistic distributions cannot be used, instead this function is moved into the integration variables through an appropriate variable substitution. In the following, we will show how to reformulate the problem of optimally sampling $\rho\left(\boldsymbol{\omega}_{i}, \boldsymbol{\omega}_{o}\right)\left(\boldsymbol{\omega}_{i} \cdot \mathbf{n}\right)$ in the context of QMC integration, starting from a uniform point set distribution.

Eq. (5) can be developed as follows:

$$
L_{o}=\int_{0}^{2 \pi} d \phi \int_{0}^{\pi / 2} \rho\left(\omega_{i}, \omega_{o}\right) L_{i}(\theta, \phi) \cos \theta \sin \theta d \theta
$$

where $\theta$ and $\phi$ are the spherical coordinates of the incident direction $\omega_{i}$ w.r.t. the z-axis.

In the case of Phong glossy BRDF:

$$
\rho\left(\omega_{i}, \omega_{o}\right)=k \frac{\left(\max \left[0,\left(\omega_{i} \cdot \omega_{r}\right)\right]\right)^{n}}{\omega_{i} \cdot \mathbf{n}}
$$

where $\omega_{r}=2\left(\omega_{0} \cdot \mathbf{n}\right)-\omega_{o}$ is the perfect mirror incident direction. A diffuse BRDF can be seen as a special case for which $\omega_{r}=\mathbf{n}$ and $n=1$ (its albedo is then $\pi k$ ).

Considering that the incident radiance function is zero for incident directions below the tangent plane (i.e. $L_{i}\left(\omega_{i}\right)=0$ if $\left.\left(\omega_{i} \cdot \mathbf{n}\right)<0\right)$, we can take the hemisphere $\Omega_{2 \pi}^{(r)}$ centered about $\omega_{r}$ as the integration domain. Our coordinate frame will then be rotated such that its $z$ is axis aligned with $\omega_{r}$ and therefore, the polar angle $\theta$ of a point $\omega$ on $\Omega_{2 \pi}^{(r)}$ will be defined by $\theta=\arccos (z)$ with $z=\left(\omega \cdot \omega_{r}\right)$.

Consequently, by making the variable substitution $z=\cos \theta$, Eq. (6) can be written as follows:

$$
L_{o}\left(\omega_{o}\right)=k \int_{0}^{2 \pi} d \phi \int_{0}^{1} L_{i}(z, \phi) z^{n} d z
$$

(c) 2013 The Author(s)

(c) 2013 The Eurographics Association and Blackwell Publishing Ltd.
Making the substitution $z^{\prime}=z^{n+1}$, we have:

$$
L_{o}\left(\omega_{o}\right)=\frac{k}{n+1} \int_{0}^{2 \pi} d \phi \int_{0}^{1} L_{i}^{\prime}\left(z^{\prime}, \phi\right) d z^{\prime}
$$

where $L_{i}^{\prime}\left(z^{\prime}, \phi\right)=L_{i}\left(z^{1 /(n+1)}, \phi\right)$. As the integral bounds still define an hemispherical integration domain, an estimate of $L_{o}\left(\omega_{o}\right)$ is obtained using Eq (1):

$$
\tilde{L}_{o}\left(\omega_{o}\right)=\frac{2 \pi k}{N(n+1)} \sum_{j=1}^{N} L_{i}\left(z_{j}^{1 /(n+1)}, \phi_{j}\right)
$$

where $\left(z_{j}, \phi_{j}\right)$ are the coordinates of a uniformly-distributed samples set $P_{N}$ on $\Omega_{2 \pi}^{(r)}$. Eq. (7) means that incident radiance function $L_{i}()$ is sampled with a sampling pattern obtained by morphing the $z$ coordinates of the samples of the uniformlydistributed set $P_{N}$ with the function $f(z)=z^{1 /(n+1)}$.

To sum up, the above derivations show how to use a spherical uniform point set to compute an approximation of the illumination integral while taking into account the BRDF shape. Although the original sample set $P_{N}$ undergoes a morphing operation, the w.c.e. of the estimate given by Eq. (7) is still strongly dependent on the characteristics of $P_{N}$ (and in particular on the energy $E_{N}$ ), as will be seen in the following sections.

\section{Tested point sets}

In this section, our goal is to compare the properties of the presented spherical Fibonacci point sets with those of the sample sets produced by the following algorithms:

- Sobol $(0,2)$-sequence with random digit scrambling as described in [KK02];

- Periodic blue noise, generated with the state of the art algorithm of de Goes et al. [dGBOD12];

- Larcher-Pillichshammer points [LP01] with random digit scrambling as described in [KK02].

Henceforth, we will refer to these three algorithms as Sobol, BNOT and L-P respectively. Fig. 2 shows different projections of sets of 512 samples generated using Sobol, BNOT and L-P, as well as an example of a spherical Fibonacci point set. We used two different techniques for projecting the unit square point sets to the unit hemisphere: the well known Lambert cylindrical projection (e.g., see [ABD12]) and the concentric map of Shirley and Chiu [SC97]. Note that these projections do not apply to Fibonacci point sets since they are generated directly in the sphere. The pattern generated by the Sobol sequence is apparently non-optimal in terms of discrepancy, since the distance of a sample to its closest neighbour is quite variable. This can be observed both on the unit square and on the unit hemisphere projections. On the other hand, the BNOT and L-P sampling patterns (Fig. 2(b) and (c) respectively), seem to be more uniformly distributed than the Sobol sequence. 


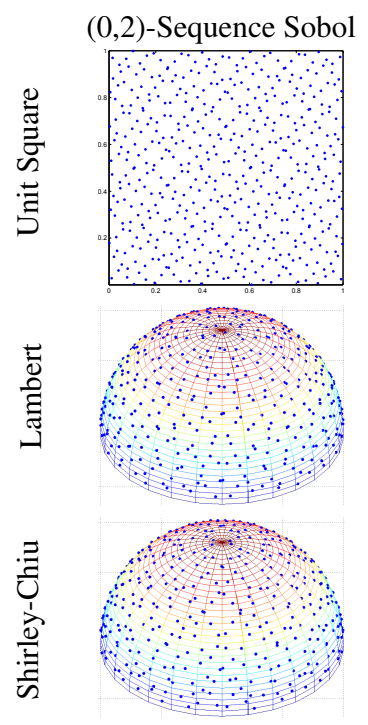

(a)

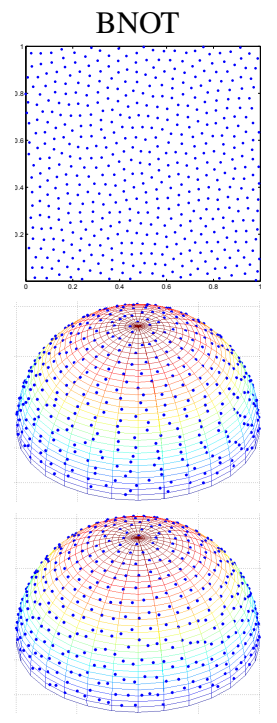

(b)

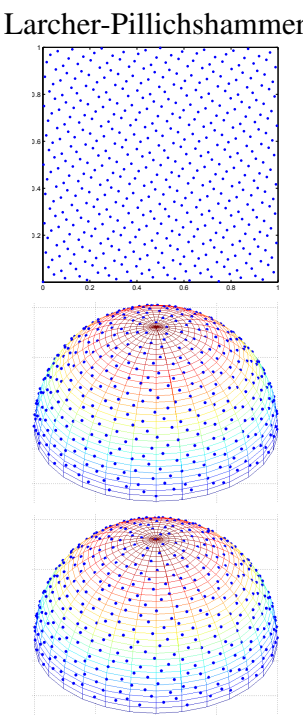

(c)

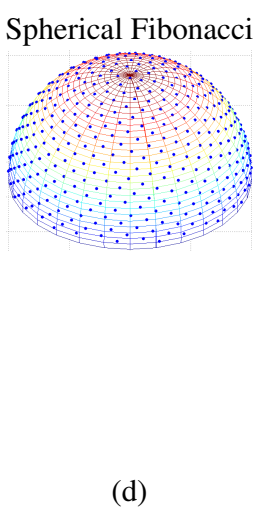

Figure 2: Examples of point sets of size 512 produced by different algorithms. Top row: unit square projection. Second row: Lambert cylindrical projection. Third row: Shirley-Chiu concentric maps projection. At the right is the Fibonacci point set generated directly in the spherical domain.

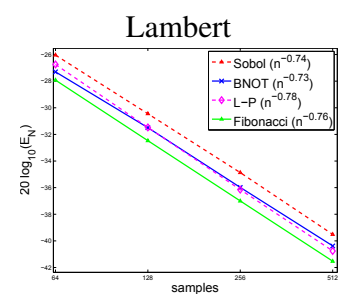

(a) Energy

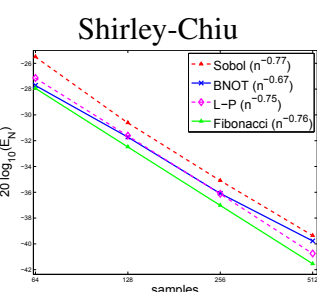

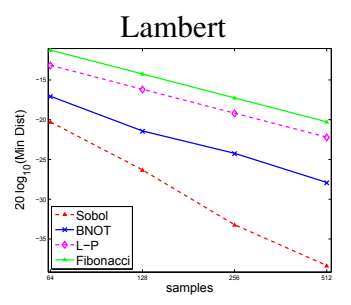

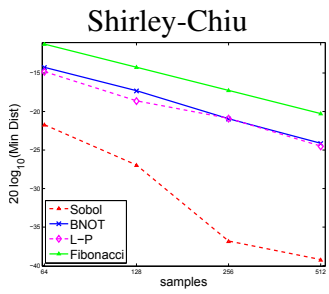

(b) Minimum inter-samples distance.

Figure 3: Properties of the tested point sets. For each metric (energy and minimum distance), the same point set was projected using the Lambert (left) and Shirley-Chiu (right) projections, except for the Fibonacci point set which is generated directly in the spherical domain.

As for the spherical Fibonacci point set (Fig. 2(d)), it exhibits superior uniformity properties when compared to all the other point sets projected on the hemisphere.

A quantitative analysis of these visual impressions can be made by comparing the different sampling patterns in terms of the energy metric defined in Eq. (3). Let us recall that, as stated in section 2.1, the w.c.e. is proportional to energy under a $C_{0}$ continuity assumption for the integrand. Fig. 3(a) clearly illustrates that the Fibonacci point set exhibits a lower energy (Eq. (3)) than the other tested algorithms and is thus expected to yield a lower w.c.e. value. In the same line of results, Fig. 3(b) shows that the minimum inter-sample distance is consistently larger for the Fibonacci point sets, which is an indication of better uniformity properties. All the tested point sets (except for BNOT using the Shirley-Chiu projection) yield approximately the same $O\left(N^{-3 / 4}\right)$ rate of decay for $E_{N}$, which corresponds to the optimum rate of convergence for the w.c.e., as explained in section 2.1. Recall that this convergence rate is obtained under a $C_{0}$ continuity assumption of the integrand, which is in general not fulfilled for illumination integrals. Nevertheless, as will be seen in the next section, these inconsistencies have marginal effects. In particular, we will show experimentally that the accuracy of the estimates given by Eq. (7) strongly depends on the energy $E_{N}$ of the uniformlydistributed samples set $P_{N}$.

\section{Results}

\subsection{General considerations}

The results presented in this section have been generated with the Mitsuba raytracer [Jak10] on a 64-bit machine 

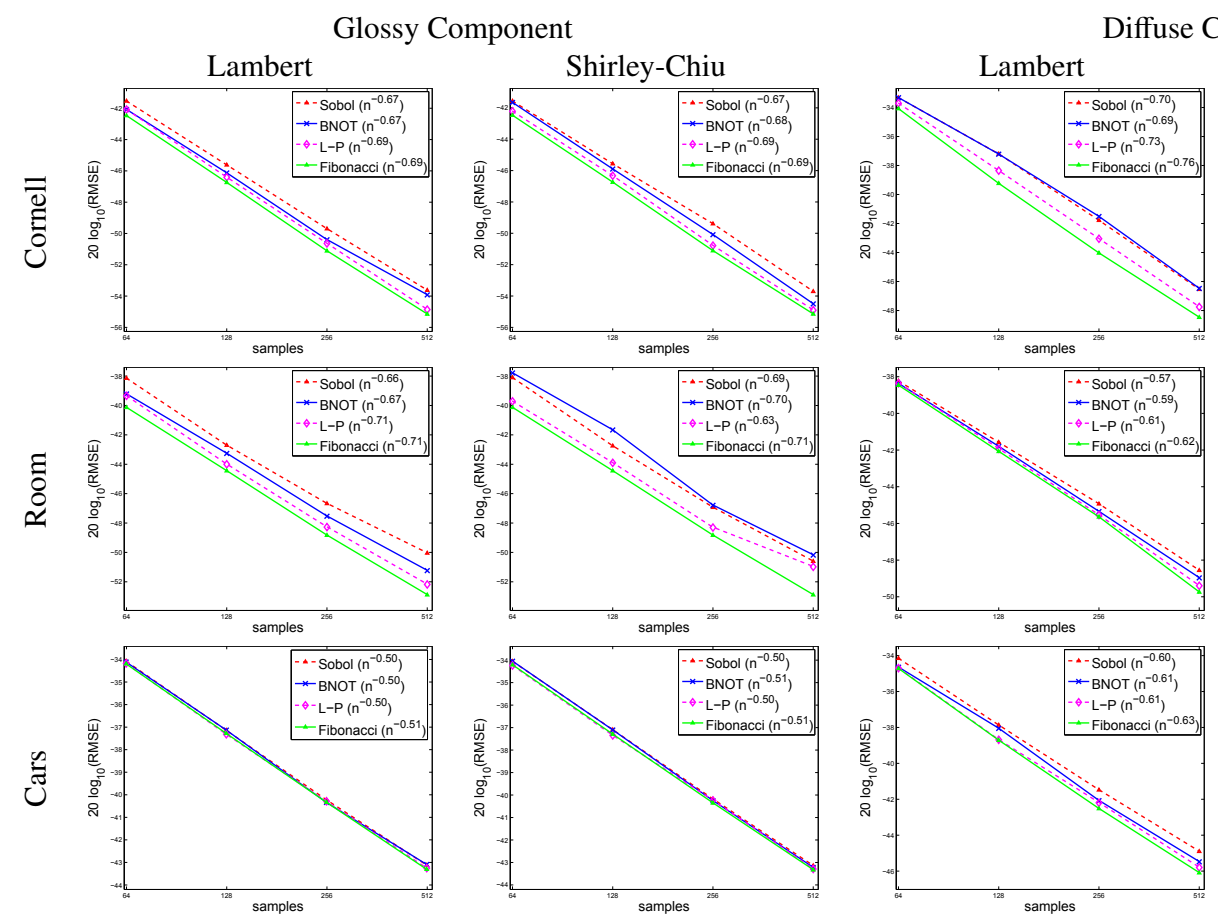

Diffuse Component
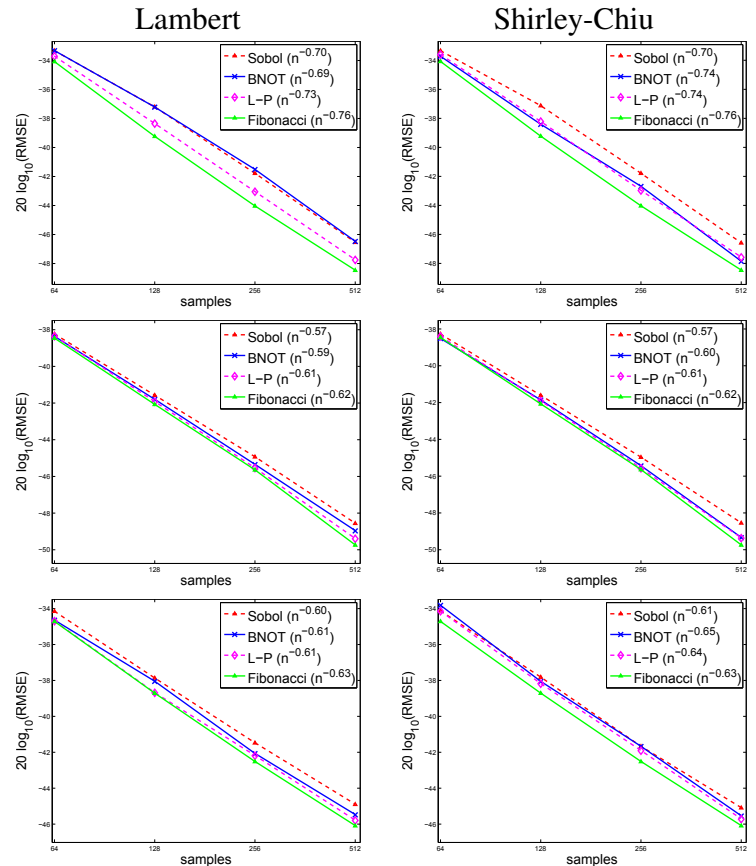

Figure 4: RMSE plots for the three test scenes as a function of the number of samples. The slopes of the RMSE line fits are displayed in the legend in-between brackets.

equipped with a $2 \mathrm{GHz}$ Intel Core $\mathrm{i} 7$ processor and a $8-\mathrm{Gb}$ RAM. Three different scenes have been used: Cornell Box (185K triangles), Room (540K triangles) and Cars (1500K triangles). The illumination integral computation has been performed in the context of final gathering for photon mapping, using the estimators given in section 4 . We have compared the results produced using the different point set construction strategies which have been presented in section 5 . A reference image has been computed using a sampling pattern produced by a Sobol sequence and a large number of samples until convergence was achieved. This reference image was then used to evaluate the RMSE of the images produced with the different point sets. For SF and BNOT scrambling is performed on the sphere (as described in section 3), but for L-P and Sobol sampling patterns it is made on the plane according to the random digit scrambling method proposed in [KK02]. We generate two distinct fixed-size sample sets for the diffuse and glossy components of the BRDF (see section 4 for details on samples set generation).

\subsection{RMSE analysis and convergence slope}

Fig. 4 shows that for the same number of samples, the spherical Fibonacci point sets yield consistently smaller RMSE values than the other tested methods. Indeed, we have not registered any case where the Fibonacci lattices have been outperformed in terms of RMSE value.
The convergence slope of QMC methods depends on the smoothness properties of the integrands. Therefore, it is not guaranteed that the theoretical convergence rate for the w.c.e. $\left(O\left(N^{-3 / 4}\right)\right.$ for $C_{0}$ continuous functions) can be obtained for highly discontinuous integrands, such as those commonly met in illumination integrals. Nevertheless, in the Cornell Box scene and in the glossy component of the Room scene, it was possible to report convergence rates close to the theoretical $O\left(N^{-3 / 4}\right)$, which means that the integrand for that scene fulfills the $C_{0}$ smoothness condition most of the times.

A comparison between the convergence rates in Fig. 4 shows that the convergence slope of the SF point set is in general as good or better than those of the other tested point sets. Note that when the convergence slope is steeper for all methods (e.g., diffuse component of the Cornell Box scene) SF point sets clearly outperform the other tested point sets. This can be explained by the fact that spherical Fibonacci point sets are more able to take advantage of smooth integrands. According to [BD11], a convergence rate as high as $O\left(N^{-2}\right)$ is possible with SF point sets in the case of very smooth integrands. On the other hand, when the rate of decay is close to $O\left(N^{-1 / 2}\right)$ (e.g., the glossy component of the Cars scene in Fig. 4), all the point sets yield similar performances since QMC in general is inefficient for very discontinuous integrands. 
R. Marques et al. / Spherical Fibonacci Point Sets for Illumination Integrals

\begin{tabular}{|c|c|c|c|c|c|c|c|c|c|}
\hline \multirow[b]{3}{*}{ Scene } & \multirow[b]{3}{*}{$\begin{array}{c}\text { Point } \\
\text { set }\end{array}$} & \multicolumn{4}{|c|}{ Glossy Component } & \multicolumn{4}{|c|}{ Diffuse Component } \\
\hline & & \multicolumn{2}{|c|}{ Lambert } & \multicolumn{2}{|c|}{ Shirley-Chiu } & \multicolumn{2}{|c|}{ Lambert } & \multicolumn{2}{|c|}{ Shirley-Chiu } \\
\hline & & RMSE & $\begin{array}{l}\text { Same quality } \\
\text { rays needed }\end{array}$ & RMSE & $\begin{array}{l}\text { Same quality } \\
\text { rays needed }\end{array}$ & RMSE & $\begin{array}{l}\text { Same quality } \\
\text { rays needed }\end{array}$ & RMSE & $\begin{array}{l}\text { Same quality } \\
\text { rays needed }\end{array}$ \\
\hline \multirow{3}{*}{$\begin{array}{l}\text { Cornell } \\
\text { Box }\end{array}$} & Sobol & $+19.2 \%$ & $658(+28.5 \%)$ & $+18.1 \%$ & $667(+30.2 \%)$ & $+24.9 \%$ & $762(+48.8 \%)$ & $+24.2 \%$ & $760(+48.5 \%)$ \\
\hline & BNOT & $+15.2 \%$ & $610(+19.2 \%)$ & $+7.8 \%$ & $601_{(+17.4 \%)}$ & $+25.9 \%$ & $785(+53.4 \%)$ & $+7.4 \%$ & $605(+18.1 \%)$ \\
\hline & L-P & $+3.4 \%$ & $544(+6.3 \%)$ & $+3.0 \%$ & $541_{(+5.6 \%)}$ & $+8.7 \%$ & $603(+17.8 \%)$ & $+10.9 \%$ & $614(+19.9 \%)$ \\
\hline \multirow{3}{*}{ Room } & Sobol & $+38.7 \%$ & $799_{(+56.0 \%)}$ & $+30.0 \%$ & $718(+40.3 \%)$ & $+14.6 \%$ & $661_{(+29.1 \%)}$ & $+14.8 \%$ & $662(+29.2 \%)$ \\
\hline & BNOT & $+20.9 \%$ & $665(+29.9 \%)$ & $+36.6 \%$ & $763(+49.1 \%)$ & $+9.4 \%$ & $601_{(+17.5 \%)}$ & $+4.8 \%$ & $569(+11.2 \%)$ \\
\hline & L-P & $+8.5 \%$ & $557(+8.8 \%)$ & $+24.6 \%$ & $665(+29.8 \%)$ & $+4.1 \%$ & $555(+8.5 \%)$ & $+4.5 \%$ & $558(+9.0 \%)$ \\
\hline \multirow{3}{*}{ Cars } & Sobol & $+2.2 \%$ & $528(+3.1 \%)$ & $+2.1 \%$ & $531(+3.7 \%)$ & $+14.7 \%$ & $634(+23.9 \%)$ & $+12.2 \%$ & $607(+18.5 \%)$ \\
\hline & BNOT & $+2.5 \%$ & $526(+2.7 \%)$ & $+1.2 \%$ & $520(+1.6 \%)$ & $+7.3 \%$ & $569(+11.0 \%)$ & $+6.4 \%$ & $559(+9.3 \%)$ \\
\hline & L-P & $+0.6 \%$ & $514(+0.4 \%)$ & $+1.1 \%$ & $518(+0.5 \%)$ & $+3.5 \%$ & $535(+4.5 \%)$ & $+4.4 \%$ & $544(+6.3 \%)$ \\
\hline
\end{tabular}

Table 1: Comparison of the results obtained using a Sobol sequence, blue noise and the Larcher-Pillichshammer points, relative to those obtained using spherical Fibonacci point sets. The glossy and diffuse components are presented separately, as well as the used projection. For each projection, the first column states the relative RMSE w.r.t. that of spherical Fibonacci, using 512 sample rays for all methods. The second column shows the number of rays required to achieve the same RMSE as spherical Fibonacci with 512 rays. In-between brackets is the corresponding percentage.

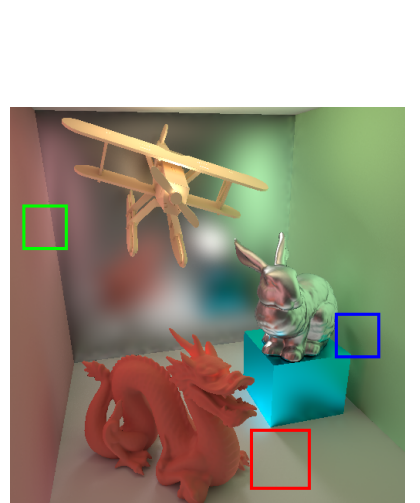

Reference $(\times 4)$

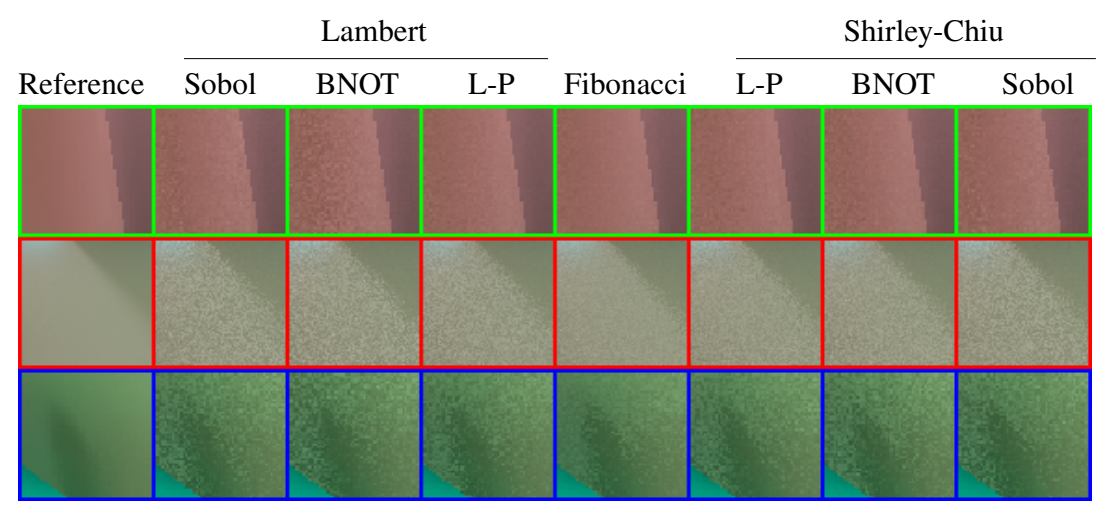

Close up views

Figure 5: Cornell Box scene (indirect radiance component only). The rabbit, the blue box and the back wall material contain a glossy BRDF, while the rest of the objects have a perfectly diffuse BRDF. Left: reference image multiplied by a factor of 4 . Right: close up views for all the used methods with 128 and 256 sample rays for the glossy and diffuse components respectively.

\subsection{Efficiency and image quality}

The benefit of using spherical Fibonacci point sets is thoroughly assessed in Tab. 1. The results show that for 512 samples per shading point, the RMSE of L-P, BNOT and Sobol point sets w.r.t. to that of SF can be up to $+8.7 \%$, $+36.6 \%$ and $+38.7 \%$ respectively. Note that this results in an even higher percentage of saved rays. As an example, for the same cases pointed out above, $\mathrm{L}-\mathrm{P}$ needs $+17.8 \%$ sample rays, BNOT $+49.1 \%$ and Sobol $+56 \%$ to achieve the same RMSE as spherical Fibonacci with 512 sample rays.

The number of rays needed to close the gap between the RMSE of SF with 512 rays and that of the other methods de- pends on the rate of convergence for the given configuration: scene, sampling method, spherical projection and radiance component. This can be clearly seen in Tab. 1 for the Room scene using the L-P points and a Lambert projection. In this case, the relative RMSE of the glossy component $(+8.5 \%)$ is more than twice that of the diffuse component $(+4.1 \%)$, using 512 samples. However, both components require approximately the same number of samples (arround 556) to achieve the same RMSE than SF with 512 samples. The reason for this is that the glossy component converges faster than the diffuse component (see legend Fig. 4).

The improvement brought by the use of spherical Fibonacci point sets can be appreciated on the close-up views 


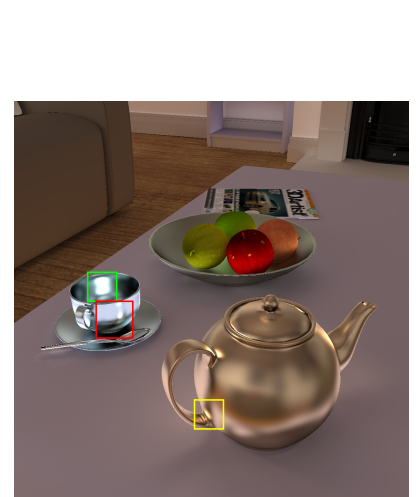

Reference $(\times 4)$

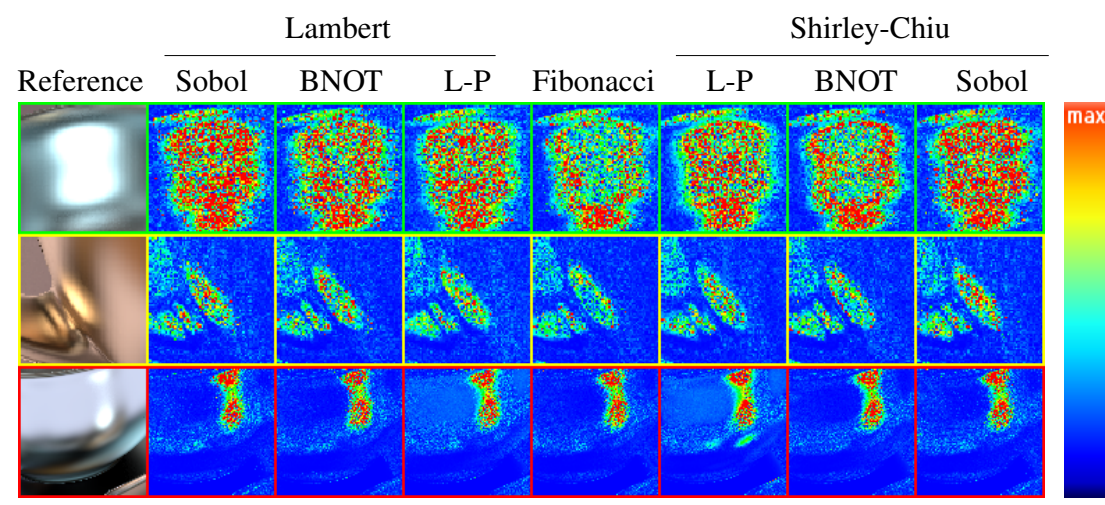

Close up views

Figure 6: Room scene (indirect radiance component only). The teapot, the teacup and the fruit-dish materials contain a glossy $B R D F$, while the rest of the objects have a perfectly diffuse BRDF. Left: reference image multiplied by a factor of 4. Right: close up views comparison of the error images for all the used methods using 32 and 128 sample rays for the glossy and diffuse components respectively. The color encodes the error magnitude.

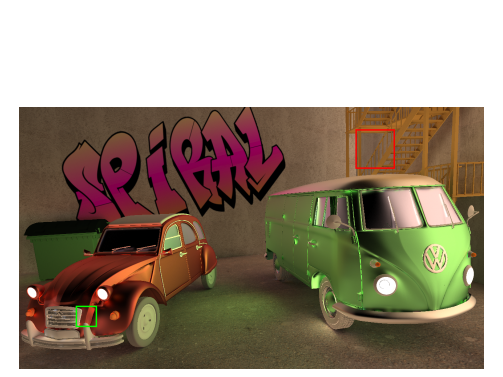

Reference $(\times 4)$

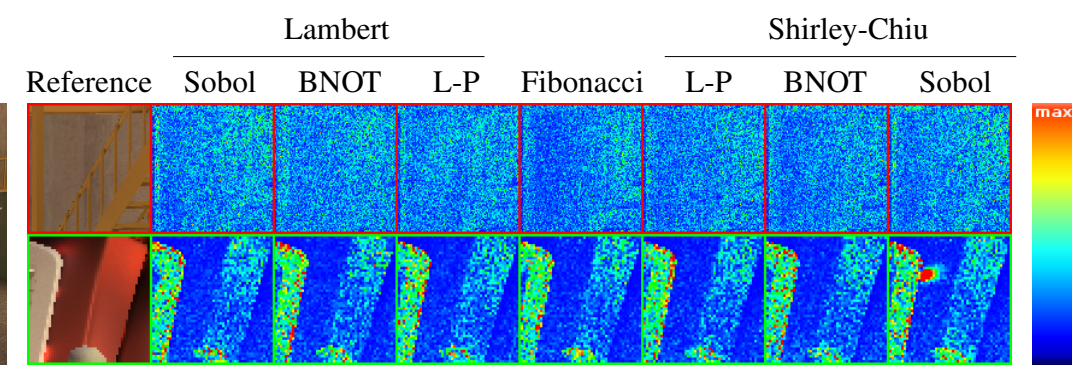

Close up views

Figure 7: Cars scene. The materials associated with the glasses and the bodyworks of both cars contain a glossy BRDF, while the rest of the objects have a perfectly diffuse BRDF. Left: reference image multiplied by a factor of 4. Right: close up views comparison of the error images for all the used methods with 512 sample rays. The color encodes the error magnitude.

of Fig. 5 which show that SF yields less visual noise compared to the other methods. As for the Room scene in Fig. 6, the error images indicate that SF performs better in critical areas such as the specular highlights. In the Cars scene (Fig. 7) on the other hand, the high discontinuity of the incident radiance makes the performance of all methods be roughly similar (as seen in Tab. 1). Nevertheless, it is still possible to identify image regions where the incident radiance is smoother, which favors SF point sets as shown on the top row of the close up views of Fig. 7.

Fig. 1 shows images computed with the SF point sets and L-P with a Lambert projection. We have compared SF with L-P since they both provide the smallest RMSE. The scene is made up of four plates, each one having a different shininess coefficient. It contains seven light sources of variable size and variable radiance producing a direct incident radiance along the plates of variable frequency. With this scene, our objective is to show how SF behaves compared to L-P point sets when the incident light contains structured circu- lar patterns and/or high frequencies that could interfere with the regular sampling pattern of SF. Despite the regularity of the SF point sets no regular patterns can be seen thanks to the used spherical scrambling method.

\section{Conclusions}

In this paper, we have presented an algorithm for efficient generation of high quality spherical QMC sequences for approximating illumination integrals. The advantages of our approach can be summarized as follows:

Simplicity: The SF point sets algorithm is simpler to implement than the other tested QMC sample sets.

Compactness: A single sequence is needed to synthesize an image. This is achieved by exploiting the axial symmetry of the BRDF lobes, which allows scrambling the point sets directly on the spherical domain using just a random axial rotation. This feature might make SF point sets particularly well-suited to GPU implementations. 
Efficiency: SF point sets outperform L-P, Sobol and blue noise-based QMC in all the test cases, allowing to save a very significant amount of sampling rays for the same image quality.

The main reason for the improvement brought by spherical Fibonacci point sets is that they better suit the spherical geometry. The other methods, in contrast, by focusing on the unit square distribution, introduce boundaries that do not exist on the sphere. Instead, our approach tries to obtain the best samples distribution directly on the sphere and then mask the effect of its regularity on the rendered image by an appropriate scrambling method.

\section{Future work}

An obvious research line is to develop adaptive sampling schemes while keeping the high quality of the energy criterion exhibited by the spherical Fibonacci point sets. As for increasing the quality of QMC BRDF-based sampling, we consider that we are already quite close to optimality and few margin for improvement exists. To go further, one could resort to non-frequentist approaches, i.e. Bayesian Monte Carlo $\left[\mathrm{BBL}^{*} 09\right]$, which allow adapting the sampling patterns according to a global covariance function of the incident radiance samples. Another research line is the reduction of the perceived error by introducing some correlation between the random rotation angles assigned to sample sets used in illumination integrals of neighbour pixels.

\section{Acknowledgments}

This work is partially funded by National Funds through the FCT - Fundação para a Ciência e a Tecnologia (Portuguese Foundation for Science and Technology) within project PEst-OE/EEI/UI0752/2011.

\section{References}

[ABD12] Aistleitner C., Brauchart J., Dick J.: Point sets on the sphere $\mathbb{S}^{2}$ with small spherical cap discrepancy. Discrete $\mathcal{E}$ Computational Geometry (2012), 1-35.

[BBL*09] Brouillat J., Bouville C., Loos B., Hansen C. D., Bouatouch K.: A bayesian monte carlo approach to global illumination. Comput. Graph. Forum 28, 8 (2009), 2315-2329.

[BD11] Brauchart J. S., Dick J.: Quasi-monte carlo rules for numerical integration over the unit sphere $\mathbb{S}^{2}$. ArXiv e-prints (Jan. 2011). http://arxiv.org/abs/1101.5450.

[Bec84] BEck J.: Sums of distances between points on a sphere - an application of the theory of irregularities of distribution to discrete geometry. Mathematika 31, 1 (June 1984), 33-41.

[BSSW12] Brauchart J. S., Saff E. B., Sloan I. H., WomersLEY R. S.: QMC designs: optimal order Quasi Monte Carlo Integration schemes on the sphere. ArXiv e-prints (Aug. 2012). http: //arxiv.org/abs/1208.3267.

[CP76] Cranley R., Patterson T.: Randomization of number theoretic methods for multiple integration. SIAM Journal on Numerical Analysis 13, 6 (1976), 904-914.
[CYC*12] Chen Z., Yuan Z., Choi Y.-K., Liu L., Wang W.: Variational blue noise sampling. IEEE Trans. Vis. Comput. Graph. 18, 10 (2012), 1784-1796.

[dGBOD12] de Goes F., Breeden K., Ostromoukhov V., Desbrun M.: Blue noise through optimal transport. ACM Trans. Graph. (SIGGRAPH Asia) 31 (2012).

[EPM*11] Ebeida M. S., Patney A., Mitchell S. A., Davidson A., Knupp P. M., Owens J. D.: Efficient maximal poisson-disk sampling. ACM Transactions on Graphics 30, 4 (2011).

[Fat11] FatTal R.: Blue-noise point sampling using kernel density model. ACM SIGGRAPH 2011 papers 28, 3 (2011), 1-10.

[GKP94] Graham R. L., Knuth D. E., Patashnik O.: Concrete Mathematics: A Foundation for Computer Science, 2nd ed. Addison-Wesley Longman Publishing Co., Inc., Boston, MA, USA, 1994.

[Gon10] González L.: Measurement of areas on a sphere using Fibonacci and Latitude-Longitude lattices. Mathematical Geosciences 42 (2010), 49-64.

[HN04] Hannay J. H., Nye J. F.: Fibonacci numerical integration on a sphere. Journal of Physics A: Mathematical and General 37, 48 (2004), 11591.

[Jak10] JАКов W.: Mitsuba renderer, 2010. http://www.mitsubarenderer.org.

[Kel12] Keller A.: Quasi-Monte Carlo image synthesis in a nutshell, 2012. https://sites.google.com/site/qmcrendering/.

[KK02] Kollig T., Keller A.: Efficient multidimensional sampling. Comput. Graph. Forum 21, 3 (2002), 557-563.

[KN06] KuIPERS L., NiederReiter H.: Uniform Distribution of Sequences. Dover Books on Mathematics. Dover Publications, 2006.

[KPR12] Keller A., Premoze S., Raab M.: Advanced (quasi) monte carlo methods for image synthesis. In ACM SIGGRAPH 2012 Courses (New York, NY, USA, 2012), SIGGRAPH '12, ACM, pp. 21:1-21:46.

[LD08] Lagae A., Dutré P.: A comparison of methods for generating Poisson disk distributions. Computer Graphics Forum 27, 1 (March 2008), 114-129.

[LP01] Larcher G., Pillichshammer F.: Walsh series analysis of the 12-discrepancyof symmetrisized point sets. Monatshefte für Mathematik 132, 1 (2001), 1-18.

[LPS86] Lubotzky A., Phillips R., Sarnak P.: Hecke operators and distributing points on the sphere I. Communications on Pure and Applied Mathematics 39, S1 (1986), S149-S186.

[NH94] NiederReiter H., H. S. I.: Integration of nonperiodic functions of two variables by Fibonacci lattice rules. Journal of Computational and Applied Mathematics 51, 1 (1994), 57 70

[Nie88] Niederreiter H.: Low-discrepancy and low-dispersion sequences. Journal of Number Theory 30, 1 (1988), 51 - 70.

[Nye03] NyE J.: A simple method of spherical near-field scanning to measure the far fields of antennas or passive scatterers. Antennas and Propagation, IEEE Transactions on 51, 8 (aug. 2003), $2091-2098$

[SC97] ShIRley P., ChIU K.: A low distortion map between disk and square. J. Graph. Tools 2, 3 (Dec. 1997), 45-52.

[SEB08] Shirley P., Edwards D., Boulos S.: Monte carlo and quasi-monte carlo methods for computer graphics. In Monte Carlo and Quasi-Monte Carlo Methods 2006, Keller A., Heinrich S., Niederreiter H., (Eds.). Springer Berlin Heidelberg, 2008, pp. $167-177$. 
[SJP06] Swinbank R., James Purser R.: Fibonacci grids: A novel approach to global modelling. Quarterly Journal of the Royal Meteorological Society 132, 619 (2006), 1769-1793.

[SK97] SAFF E., KuIJlaARs A.: Distributing many points on a sphere. The Mathematical Intelligencer 19 (1997), 5-11.

[Sob67] Soвol I.: On the distribution of points in a cube and the approximate evaluation of integrals. USSR Computational Math. and Math. Phys. 7 (1967), 86-112.

[Sve94] Svergun D. I.: Solution scattering from biopolymers: advanced contrast-variation data analysis. Acta Crystallographica Section A 50, 3 (May 1994), 391-402.

[Vog79] Vogel H.: A better way to construct the sunflower head. Mathematical Biosciences 44, 3-4 (1979), 179-189. 